\title{
Mapping Tissue Optical Attenuation to Identify Cancer Using Optical Coherence Tomography
}

\author{
Robert A. McLaughlin ${ }^{1}$, Loretta Scolaro ${ }^{1}$, Peter Robbins ${ }^{2}$, Christobel Saunders ${ }^{3,4}$, \\ Steven L. Jacques ${ }^{5}$, and David D. Sampson ${ }^{1}$ \\ ${ }^{1}$ Optical+Biomedical Engineering Laboratory, Uni. Western Australia, WA, Australia \\ robertm@ee. uwa . edu . au \\ ${ }^{2}$ PathWest QEII Medical Centre, WA, Australia \\ ${ }^{3}$ Sir Charles Gairdner Hospital, WA, Australia \\ ${ }^{4}$ School of Surgery, Uni. Western Australia, WA, Australia \\ ${ }^{5}$ Biomedical Engineering/Dermatology, Oregon Health \& Science University, OR, USA
}

\begin{abstract}
The lymphatic system is a common route for the spread of cancer and the identification of lymph node metastases is a key task during cancer surgery. This paper demonstrates the use of optical coherence tomography to construct parametric images of lymph nodes. It describes a method to automatically estimate the optical attenuation coefficient of tissue. By mapping the optical attenuation coefficient at each location in the scan, it is possible to construct a parametric image indicating variations in tissue type. The algorithm is applied to ex vivo samples of human axillary lymph nodes and validated against a histological gold standard. Results are shown illustrating the variation in optical properties between cancerous and healthy tissue.
\end{abstract}

\section{Introduction}

Cancer is the second most common cause of death worldwide, accounting for $12.5 \%$ of all mortality [1]. During cancer surgery, a critical task is to assess the spread (metastasis) of the cancer. The lymphatic system is a common route for metastasis. It is typically assessed by excising lymph nodes near the tumor and performing histo-pathological analysis. However, this results in the unnecessary excision of many healthy, uninvolved lymph nodes and can result in chronic disruption to the lymphatic system. For example, in breast cancer surgery, approximately $26 \%$ of patients undergoing local lymph node removal (axillary clearance) will suffer lymphedema.

Optical coherence tomography (OCT) [2] is a high-resolution imaging modality. It is conceptually similar to ultrasound, but uses reflections of low-power, near-infrared light instead of sound waves. It is capable of imaging tissue with a resolution of approximately 10 microns, and has the potential to assess lymph node involvement in cancer in vivo [3]. However, the signal values in an OCT data set are not determined absolutely by the tissue. They are also a function of many imaging and experimental parameters, including tissue depth, power and incident angle of the light source, imaging optics, and the effects of overlying tissue, including shadowing and refraction. For this reason, it is difficult to differentiate between image features due to the optical properties of a particular tissue type, and those due to imaging artifacts. 
In this paper, we describe a method to differentiate tissues with differing optical properties in an OCT scan. Results are presented visually, as a parametric image in which intensity indicates the relative optical attenuation coefficient at a particular location. To the best of our knowledge, this is the first time that a parameterized OCT image has been generated in this manner. We present results from both phantom data, and cancerous and non-cancerous human lymph nodes.

\section{Methods}

\subsection{Optical Properties of Tissue}

OCT acquires images of tissue structure by detecting the coherence-gated backscattering of near infrared light. However, tissue is an optically turbid media and the signal attenuates and spreads with increasing depth, limiting the imaging depth of OCT to approximately $2-3 \mathrm{~mm}$. In OCT image formation, a focused light source is directed onto a particular location on the tissue and a depth scan is acquired in the $z$ direction. Using terminology borrowed from ultrasound, this one-dimensional scan is referred to as an A-scan. A three-dimensional data volume is constructed by acquiring a sequence of A-scans at different $x, y$ locations over the area to be scanned.

Attenuation of the OCT signal can be modeled using Beer's Law. The signal intensity $I(z)$ at a depth $z$ can be expressed as follows [4]:

$$
I(z)=I_{0} e^{-\mu z} R(z) e^{-\mu z}=I_{0} e^{-2 \mu z} R(z)
$$

where $I_{0}$ is the incident intensity, $R(z)$ is the depth-dependent reflectivity, and $\mu$ is the observed attenuation coefficient of the tissue. The factor of 2 arises because the light must both travel into the tissue and return to the detector. The attenuation coefficient $\mu$ is affected by both scattering and absorption [5,6], parameterized by the scattering coefficient $\mu_{s}$ and absorption coefficient $\mu_{a}$.

Scattering refers to the lossless redirection of light due to a local change in refractive index. For example, this may be due to interaction with structures such as cell membranes, collagen fibrils or cell nuclei [7]. The angular distribution of scattering may be modeled with a Henyey-Greenstein function, parameterized by an anisotropy factor $g$ [8]. Let $\theta$ be the deviation in angle of light after a scattering event. Then $g$ is defined as the expected value of $\cos \theta$. A value of $g \approx 1$ indicates forward scattering events in which light will continue in roughly the same direction. A value of $g \approx 0$ indicates an isotropic scattering function, where the angle of scatter is equally likely in all directions. Tissue is typically strongly forward scattering, with values of $g$ in the range 0.85 to 0.97 [9].

The observed attenuation coefficient $\mu$ may be modeled by the following equation:

$$
\mu=2\left[a(g) \mu_{s}+\mu_{a}\right] G
$$

where:

$$
a(g)=1-e^{-\frac{(1-g)^{m}}{n}}
$$


with $m=0.6651$ and $n=0.1555$ [10]. The factor $a(g)$ modifies $\mu_{s}$ to represent the effectiveness of scatter in image formation and ranges from 1 at low $g$, to 0 at $g=1$. For $g=0.97, a(g) \approx 0.46$ and $\mu_{s}$ is half as effective in preventing photons from reaching the coherence gate. For $g=0.85, a(g) \approx 0.84$. For the $1325 \mathrm{~nm}$ central wavelength of light used in this report, the ratio of wavelength to scattering particle size in the tissue is greater than for visible or shorter near-infrared wavelengths. Hence, the value of $g$ is likely to be about $0.4-0.6$. Therefore, $a(g)$ is probably greater than 0.95 . The factor $G$ is the extra photon path length as photons are delivered obliquely toward a focus. With the low numerical aperture used in OCT this factor is approximately unity.

For near infrared light in tissue, scattering is the dominant attenuation mechanism [11], and the scattering coefficient $\mu_{s}$ is typically a factor of 10-100 times larger than the attenuation coefficient $\mu_{a}$ [12]. For the $1325 \mathrm{~nm}$ wavelength used in the experiments of this report, $\mu_{a}$ for pure water is $0.149 \mathrm{~mm}^{-1}$. The value of $\mu_{s}$ for tissues at $1325 \mathrm{~nm}$ is in the $5-10 \mathrm{~mm}^{-1}$ range. Thus, the absorption can be neglected, and since $G \approx 1$, the equation for attenuation may be approximated by:

$$
\mu \approx 2 a(g) \mu_{s}
$$

Assuming that a single A-scan intersects only a single type of tissue, the attenuation parameter $\mu$ may be used to characterize a tissue. By taking the logarithm of the OCT signal values along a single A-scan, $\mu$ can be extracted from the slope of the line of best fit. We calculate this slope for each $x, y$ location in the data set, deriving a $2 \mathrm{D}$ map $\mu(x, y)$. This $\mu$ is then represented visually as a 2D parametric image of the tissue in which the $\mu(x, y)$ is indicative of tissue type.

\section{Experiment}

Phantom and human ex vivo tissue samples were scanned with a swept-source OCT system (Thorlabs, New Jersey, USA) with a central wavelength of $1325 \mathrm{~nm}$ and a spectral bandwidth of $100 \mathrm{~nm}$. The transverse resolution was $15 \mu \mathrm{m}$ and axial resolution was $12 \mu \mathrm{m}$ in air.

\subsection{Phantom Experiment}

Two imaging phantoms [13] were prepared to mimic the optical properties of tissue. Titanium oxide powder (Sigma Aldrich, St. Louis, USA), with an average particle size of $5 \mu \mathrm{m}$, was mixed into a base material of room temperature vulcanizing (RTV) silicone (Wacker, Munich, Germany). Different quantities of titanium oxide were used to vary the optical properties of the phantoms, with the second phantom containing three times the concentration of the first. Both phantoms were scanned using OCT and the relative attenuation coefficient was estimated by finding the slope of the line of best fit to the log intensity values along an A-scan.

\subsection{Human Tissue Experiment}

Three human breast lymph nodes were taken from patients undergoing axillary clearance. Fresh excised lymph tissue was dissected into $2 \mathrm{~mm}$ slices and a 3D-OCT scan 
of the fresh tissue was acquired. Each sample underwent subsequent histological analysis using Haematoxylin and Eosin $(\mathrm{H} \& \mathrm{E})$ staining, and revealed the first node to be healthy, the second node to contain a well defined cluster of metastasis, and the third node to contain diffuse malignant cells intermingled throughout the tissue.

\subsection{Tissue Image Pre-processing}

During OCT scanning of the lymph node samples, the tissue was laid on a glass slide and immersed in a small quantity of glycerol to match the refractive index of the glass in order to reduce signals from the glass-air-tissue interfaces. However, tissue samples typically do not sit flat upon the glass slide, resulting in small glycerol-filled gaps between the tissue and glass. Unfortunately, this confounds the process of automatically estimating the attenuation coefficient. In order to characterize the tissue within an A-scan, it is necessary to identify where in the A-scan the light enters the tissue. Measurements prior to this position are not indicative of the tissue. The position within the A-scan will be dependent upon the size of any gap between the glass slide and the tissue. Identifying this position manually is impractical for a typical OCT acquisition comprising over a million A-scans.

Simple image processing techniques were utilized to automatically identify the point within each A-scan where the light entered the tissue. Note that each A-scan comprises a large peak of reflectivity early in the A-scan, corresponding to the change in refractive index as the light passes from air into the glass slide. This corresponds to a local maximum in the derivative of the intensity of the A-scan and was automatically identified for all A-scans in the acquisition, defining a set of points on the glass surface (plus a number of outliers). A 2D plane was robustly fitted to this point set and the intersection of the plane with each A-scan was taken as specifying the airglass interface. Having extracted the location of the glass slide, the start of the tissue was found by averaging A-scan values within a moving window, and identifying the location at which the value exceeded a threshold. The attenuation co-efficient was then calculated by computing the slope of the line of the best fit for the log of the Ascan values, from the start of the tissue and extending over an optical path length of $0.5 \mathrm{~mm}$.

\section{Results}

Figure 1 shows representative A-scans from the two phantoms. The log of the reflectivity values are plotted against image depth. The line of best fit is superimposed upon each A-scan, and its slope is indicative of the concentration of titanium oxide powder. Note that signal attenuation is more gradual in the first phantom (Fig. 1, left) and markedly increased in the second (Fig. 1, right) with three times the concentration of titanium oxide. Fluctuations in the signals are characteristic of the speckle present in OCT, noted to be less in the phantom with a higher concentration of titanium oxide.

Figures 2-4 show parameterized OCT images of human lymph nodes with corresponding H\&E histology. The intensity at each pixel in the parameterized OCT image is indicative of the attenuation coefficient for an A-scan that extends perpendicular to the image and into the sample, calculated over an optical path length of $0.5 \mathrm{~mm}$. Fig. 2 

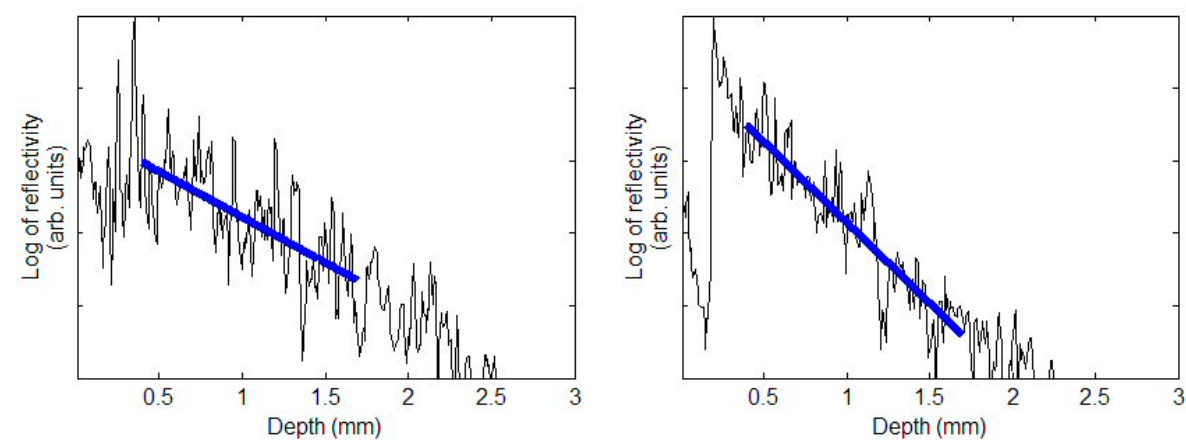

Fig. 1. A-scans from two phantom objects, with line of best fit showing attenuation due to scatter. Left: Phantom 1 (low concentration of $\mathrm{TiO}_{2}$ in silicon). Right: Phantom 2 (high concentration of $\mathrm{TiO}_{2}$ in silicon).
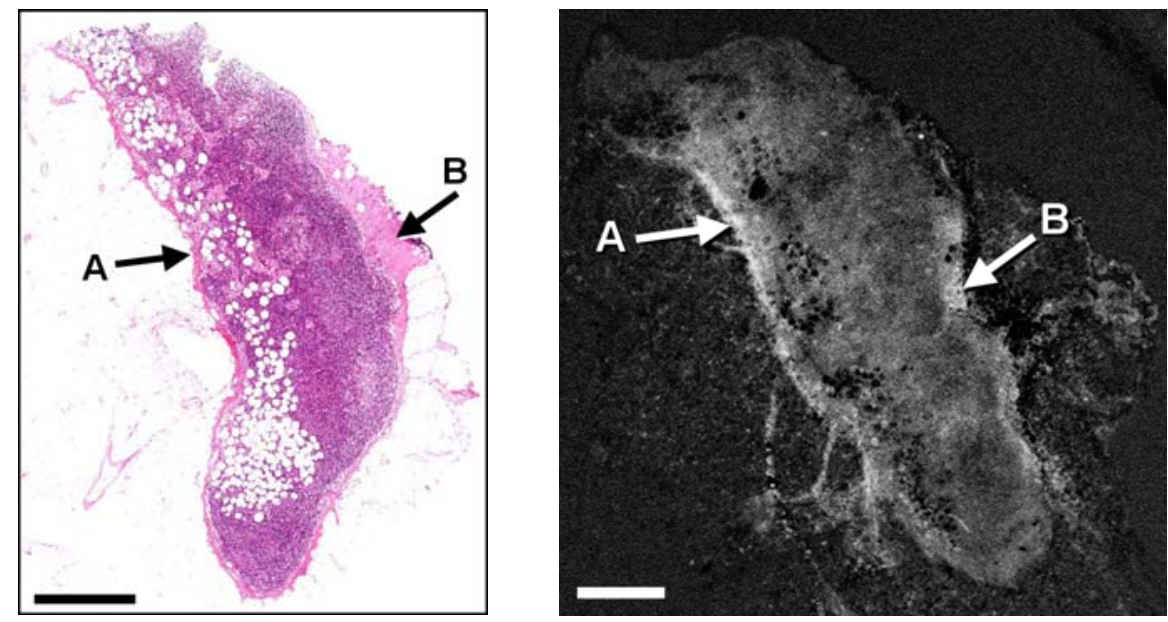

Fig. 2. Reactive, normal (uninvolved) axillary lymph node. Left: H\&E histology; Right: Parameterized OCT image. (A, B): Stroma (bright areas). Scale bar $=1 \mathrm{~mm}$.

shows a reactive, benign (i.e., uninvolved) lymph node. The sample comprises primarily of the cortex of the lymph node, bounded by the fibrous stromal tissue of the lymph node capsule. Stroma was observed to have a higher attenuation coefficient than cortex, presenting as bright areas in the image (labeled A, B).

Figure 3 shows an involved lymph node, containing a well delineated cluster of metastatic malignant cells (labeled A), visible in both the parameterized OCT image and histology. The outer cortical regions of the lymph node (labeled B, C) appear as dark areas in the parameterized OCT image (low scatter). Lighter areas typically correspond to sinuses extending through the paracortex and medulla of the node. 

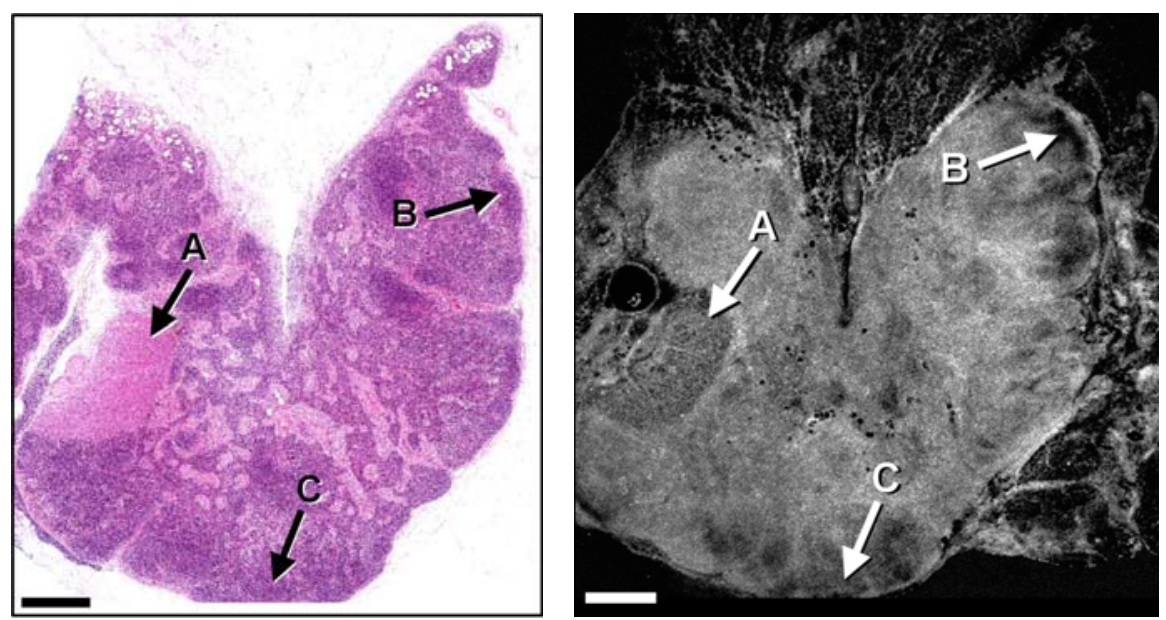

Fig. 3. Involved (malignant) axillary lymph node with well defined deposits of metastatic breast cancer. Left: H\&E histology; Right: Parameterized OCT image. (A) Metastasis; (B, C): Lymph cortex (dark areas). Scale bar $=1 \mathrm{~mm}$.
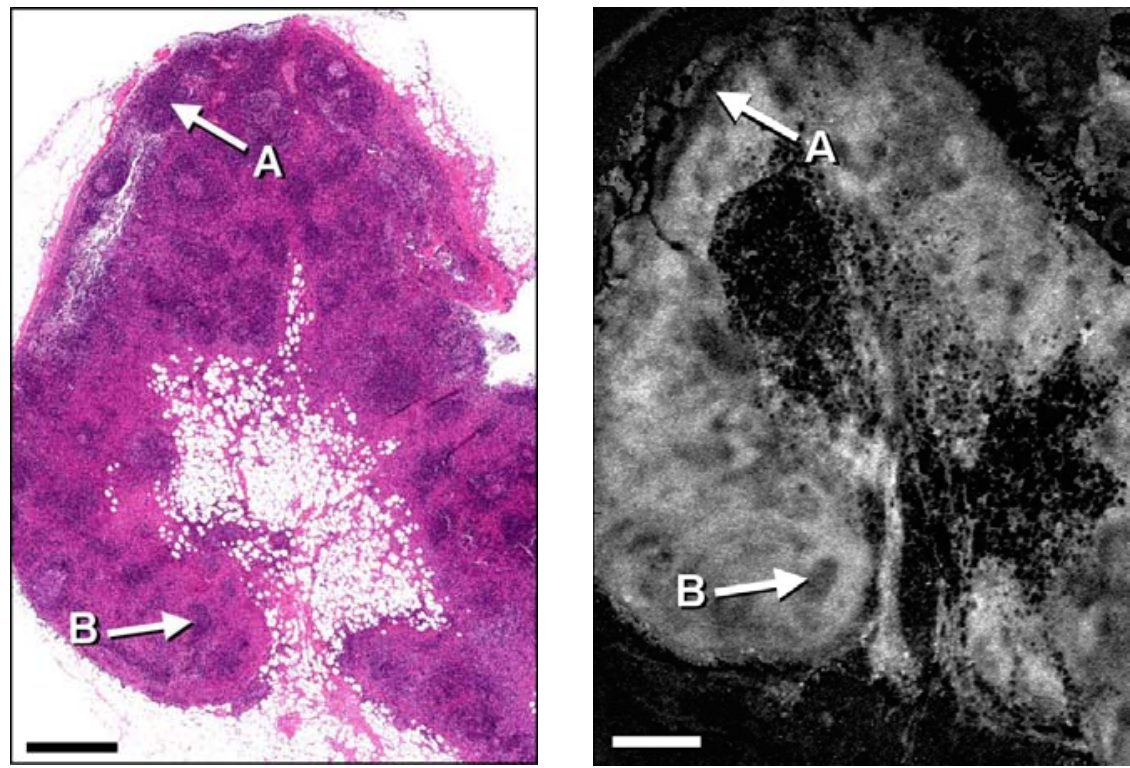

Fig. 4. Involved (malignant) axillary lymph node, with diffuse involvement of the node tissue by metastatic breast cancer cells. Left: H\&E histology; Right: Parameterized OCT image. (A, B): Residual areas of healthy cortex (dark areas). Scale bar $=1 \mathrm{~mm}$. 
Figure 4 shows an involved lymph node in which the metastatic malignant cells are distributed in a diffuse fashion within the nodal lymphoid tissue. Rather than forming discrete aggregates, the metastatic malignant cells in this example are diffusely intermingled amongst the normal cells native to the lymph node. Malignant areas (i.e., those containing the metastatic breast cancer cells) typically have a higher attenuation coefficient (light areas) than the residual uninvolved lymph cortex. Two example dark areas corresponding to residual cortex are labeled A, B.

\section{Discussion}

The results presented here demonstrate the potential of OCT to differentiate tissue types based on estimates of the tissue's attenuation coefficient. Cancerous areas were visibly distinguishable from surrounding non-cancerous tissue. Stroma tissue was seen to be highly scattering, in agreement with previously reported results [4].

The estimation of such optical properties from OCT presents a useful contrast mechanism. Importantly, it removes some of the variability based on the image acquisition settings. However, the current implementation is based on significant assumptions. The technique assumes that each A-scan samples only a single tissue type. In complex biological structures, this may not be the case and image segmentation techniques will be required to identify regions of homogeneous tissue, such that the attenuation coefficient of each tissue may be calculated separately. The current implementation also utilizes signal attenuation as the single parameter for image contrast. Other authors have used different techniques to separately estimate the effects of the scattering coefficient $\mu_{s}$ and anisotropy $g$ of scattering [10]. We note that more complex models are possible, accounting for the heterogeneity of size, shape, and density of the scatterers in biological tissue [14] and speckle noise [15], and may provide more sophisticated quantitative measures for tissue differentiation.

\section{Conclusion}

This paper has presented preliminary results for a new form of parameterized OCT image, in which the intensity of each pixel is indicative of the tissue's attenuation coefficient across an A-scan. The results demonstrate the potential of such a parameterized image to distinguish between cancerous and non-cancerous tissue. Future work will utilize segmentation techniques to account for heterogeneity in tissue type within individual A-scans.

\section{References}

1. Garcia, M., Jemal, A., Ward, E.M., Center, M.M., Hao, Y., Siegel, R.L., Thun, M.J.: Global Cancer Facts \& Figures 2007. American Cancer Society, Atlanta (2007)

2. Bouma, B.E., Tearney, G.J.: Handbook of Optical Coherence Tomography. Marcel Dekker Inc., USA (2002)

3. Luo, W., Nguyen, F.T., Zysk, A.M., Ralston, T.S., Brockenbrough, J., Marks, D.L., Oldenburg, A.L., Boppart, S.A.: Optical Biopsy of Lymph Node Morphology using Optical Coherence Tomography. Technol. Cancer Res. T 4(5), 539-547 (2005) 
4. Collier, T., Follen, M., Malpica, A., Richards-Kortum, R.: Sources of Scattering in Cervical Tissue: Determination of the Scattering Coefficient by Confocal Microscopy. Appl. Optics 44, 2072-2081 (2005)

5. Van Gemert, M.J.C., Jacques, S.L., Sterenborg, H.J.C.M., Star, W.M.: Skin Optics. IEEE Trans. Biomed. Eng. 36, 1146-1154 (1989)

6. Simpson, C.R., Kohl, M., Essenpreis, M., Cope, M.: Near-infrared Optical Properties of Ex vivo Human Skin and Subcutaneous Tissues Measured using the Monte Carlo Inversion Technique. Phys. Med. Biol. 43, 2465-2478 (1998)

7. Wang, L.V., Wu, H.: Biomedical Optics. Wiley Interscience, Malden (2007)

8. Yoon, G., Welch, A., Motamedi, M., van Gemert, M.: Development and Application of Three-dimensional Light Distribution Model for Laser Irradiated Tissue. IEEE J. Quantum Elect. 23, 1721-1733 (1987)

9. Key, H., Davies, E.R., Jackson, P.C., Wells, P.N.T.: Optical Attenuation Characteristics of Breast Tissues at Visible and Near-infrared Wavelengths. Phys. Med. Biol. 36, 579-590 (1991)

10. Samatham, R., Jacques, S.L., Campagnola, P.: Optical Properties of Mutant Versus Wildtype Mouse Skin Measured by Reflectance-mode Confocal Scanning Laser Microscopy (rCSLM). J. Biomed. Opt. 13, 041309-1-041309-7 (2008)

11. Collier, T., Arifler, D., Malpica, A., Follen, M., Richards-Kortum, R.: Determination of Epithelial Tissue Scattering Coefficient Using Confocal Microscopy. IEEE J. Sel. Top. Quant. Elect. 9, 307-313 (2003)

12. Qu, J., MacAulay, C., Lam, S., Palcic, B.: Optical Properties of Normal and Carcinomatous Bronchial Tissue. Appl. Optics 33, 7397-7405 (1994)

13. Pogue, B.W., Patterson, M.S.: Review of Tissue Simulating Phantoms for Optical Spectroscopy, Imaging and Dosimetry. J. Biomed. Opt. 11(4), 041102-1-041102-16 (2006)

14. Schmitt, J.M., Knüttel, A.: Model of Optical Coherence Tomography of Heterogeneous Tissue. J. Opt. Soc. Am. A 14, 1231-1242 (1997)

15. Schmitt, J.M., Xiang, S.H., Yung, K.M.: Speckle in Optical Coherence Tomography. J. Biomed. Opt. 4(1), 95-105 (1999) 